\title{
El Parámetro del Sujeto Nulo y la enseñanza del español como lengua extranjera. Reflexión gramatical
}

\author{
P. Pablo DEVÍS MÁRQUEZ \\ Universidad de Cádiz \\ Departamento de Filología \\ pedropablo.devis@uca.es
}

Recibido: septiembre 2010

Aceptado: marzo 2011

\section{RESUMEN}

En este artículo se aborda la aplicación del Parámetro del Sujeto Nulo en el proceso de adquisición del español como L2. Una vez detectadas las dificultades que esta aplicación entraña, se postula la importancia de la reflexión gramatical en el proceso de enseñanzaaprendizaje. Finalmente, se presenta una propuesta de reflexión gramatical que sirva de material didáctico en la enseñanza-aprendizaje del fenómeno de la presencia/ausencia del sujeto en el aula de español como L2.

Palabras clave: Enseñanza-aprendizaje del español como L2; Parámetro del Sujeto Nulo.

\section{Le Paramètre du Sujet Nul et l'enseignement de l'Espagnol comme langue étrangère. Réflexion grammaticale}

\section{RÉSUMÉ}

Dans cet article, il est question de l'application du Paramètre du Sujet Nul dans le processus d'acquisition de l'Espagnol L2. Repérées les difficultés que cette application entraîne, on insiste sur l'importance de la réflexion grammaticale dans le processus d'enseignementapprentissage. Finalement, on présente une proposition de réflexion grammaticale qui sert de matériau didactique pour l'enseignement-apprentissage du phénomène de la présence/absence du sujet dans les cours d'Espagnol L2.

Mots-clés : Enseignement-apprentissage de l'Espagnol L2; Paramètre du Sujet Nul.

The Null Subject Parameter and Teaching Spanish as a foreign language. A Grammatical reflection

\begin{abstract}
This article is about the application of the Null Subject Parameter to the acquisition process of Spanish L2. Having detected the problems that this application involves, the importance of the grammatical reflection in the teaching-learning process is proposed. Finally, we present a proposal of grammatical reflection that is useful as teaching-learning material for teachinglearning the presence/absence of the subject phenomenon in the Spanish L2 classroom.
\end{abstract}


Keywords: Teaching-learning Spanish L2; Null Subject Parameter.

SUMARIO: 1. Adquisición de lenguas y el Parámetro del Sujeto Nulo. 2. La reflexión gramatical en el proceso de enseñanza-aprendizaje. 3. Descripción lingüística y elaboración de reflexión gramatical: presencia y ausencia del sujeto en español. 4. Resultado y conclusión. Referencias bibliográficas.

\section{ADQUISICIÓN DE LENGUAS Y EL PARÁMETRO DEL SUJETO NULO}

A partir de la segunda mitad del siglo XX han sido muchos los trabajos preocupados por la adquisición -también por la enseñanza- de segundas lenguas que, en algunas de ellas, han abordado la cuestión de la elisión del sujeto con verbos conjugados en forma personal desde los presupuestos teóricos del modelo de Principios y Parámetros. ${ }^{1}$ En este sentido, ha sido frecuente a la hora de tratar este fenómeno aludir al denominado Parámetro del Sujeto Nulo y catalogar las lenguas que lo presentan -entre las que se encuentra el español- como lenguas pro-drop. ${ }^{2}$

Este tipo de trabajos se ha centrado, fundamentalmente, en tres aspectos básicos: 1) el desencadenante o causa de que el citado parámetro se encuentre en determinadas lenguas; 2) las dificultades que la presencia de este parámetro implica en la adquisición de una L2, y 3) los efectos que este parámetro provoca en la interlengua del aprendiz. En lo que al primero de estos aspectos se refiere, en un principio, se asoció la elisión del sujeto a la existencia en una lengua de una flexión verbal rica que permita la identificación del argumento elidido (cf.Perlmutter 1971, Taraldsen 1978, Chomsky ${ }^{2} 1982$, 1986, 1988a, 1988b, Rizzi 1982, Safir 1982, Hyams 1983, Liceras/Díaz 1999 y 2000, Liceras/Valenzuela/Díaz 1999, etc.). ${ }^{3}$ No obstante, el

${ }^{1}$ Acerca de este modelo, cf. Chomsky ${ }^{2} 1982,1986,1988$ a y 1995 y Chomsky y Lasnik 1993, entre otros. En lo que concierne a la adquisición de segundas lenguas desde este punto de vista, cf. Liceras 1996.

${ }^{2}$ Acerca del concepto de sujeto nulo con las formas verbales flexivas (primero PRO, luego pro, también $\emptyset$ ) en el ámbito de la gramática generativa, fundamento de lo que en la teoría de principios y parámetros es el Parámetro del Sujeto Nulo, cf., entre otros, Chomsky ${ }^{2} 1982$, 231-278 y 1988a 43, Rizzi 1982, 115-117, Suñer 1982 y 1984, Burzio 1986, 85 y ss., Jaeggli 1980 y 1982, Safir 1982, Jaeggli/Safir (eds.) 1989, etc. Frente a lenguas como el inglés, el francés o el alemán, que carecen por completo del rasgo, y otras como el finlandés y el hebreo, que lo muestran de modo parcial ( cf. Camacho 2010, 84, que cita, a su vez a Vanika/Levi 1999 y 2000), es habitual presentar el español como un ejemplo prototípico de lengua pro-drop.

${ }^{3}$ En lo que concierne al hecho de que los aprendices de español como L2 no legitiman los sujetos nulos a través de un principio de la gramática -algo que sí sucede en español cuando se adquiere como L1-, sino que simplemente -recurriendo al principio universal de la identificación- los identifican, cf. Liceras/Laguardia/Fernández/Fernández/Díaz 1998, Liceras 1994 y 1995, Liceras/Díaz/Maxwell 1998, Liceras/Díaz 1999 y 2000. Lo que hacen los 
hecho de que lenguas ricas en cuanto a la flexión verbal, caso del alemán o el islandés, no permitan la supresión del sujeto (cf. Mohanan 1983 y Huang 1984) y el de que lenguas sin esa riqueza en la flexión sí la acepten, caso del chino y el japonés (cf. Jaeggli/Hyams 1988, Jaeggli/Safir 1989 y Lu 1997) condujo a que Jaeggli/Safir 1989 postularan el Principio de Uniformidad Morfológica (PUM) como desencadenante de la existencia del Parámetro del Sujeto Nulo en una lengua determinada. Según el PUM, que una lengua se muestre uniforme en lo que se refiere a la flexión verbal, sea porque el verbo no la muestra en ningún caso (chino y japonés), sea porque siempre está presente (español), es el requisito indispensable para que pueda elidir los sujetos que acompañan a los verbos conjugados en forma personal. Que lenguas como el inglés, el francés o el alemán carezcan de esta uniformidad explica la ausencia en ellas de sujetos nulos (cf. Borer 1984, Wexler/Manzini 1987, Lebeaux 1988, Chomsky 1992, etc.).Lakshmanan 1994, 2 y ss., no obstante, cuestiona la consideración del PUM como principio universal y advierte de la falta de evidencias acerca de que los aprendices de una L2 tengan acceso al PUM. Las relaciones entre flexión verbal y sujetos nulos, piensa, no parece que jueguen un papel importante en la adquisición de una L2. Lakshmanan prefiere la propuesta de Lillo-Martin 1991 y Wang/Lillo-Martin/Best/Levitt 1992, según la cual la elisión del sujeto está relacionada con dos parámetros diferentes: el Parámetro del Sujeto Nulo y el Parámetro del Discurso Orientado.

En lo que atañe a las dificultades que el Parámetro del Sujeto Nulo supone en la adquisición de una L2, se ha discutido el carácter marcado o no de este parámetro, esto es, si la elisión del sujeto es el valor inicial en el ámbito de la Gramática Universal, por lo que se situaría en el estadio inicial del proceso de adquisición de una lengua, o si, por el contrario, representa una opción a la que sólo se tiene acceso mediante el contacto directo con los datos que muestra la lengua que se aprende. En este asunto se contraponen dos posiciones: la que asigna un carácter marcado al parámetro (cf. White 1985, 1986 y 1989, 117-139 y 141-171, Berwick 1985, Rizzi 1986) frente a la que le otorga un valor no marcado (cf. Phinney 1987, Brucart 1998). ${ }^{4}$ Decantarse por la opción del valor no

adultos es que «imponen las representaciones de la L1 sobre los datos de la L2 y los reestructuran» (cf. Liceras/Díaz 2000, 62).

${ }^{4}$ Como dato para sostener el carácter no marcado del parámetro, suele acudirse al hecho de que los niños que aprenden inglés - una lengua que no es pro-drop-como lengua materna pasan por un estadio en el que omiten sistemáticamente los sujetos (cf. Brown 1973, Bloom/Lightbown/Hood 1975, Guilfoyle 1984, Hyams 1983, 1986 y 1992). Jaeggli/Hyams 1988 y Hyams 1992 explican este hecho sobre la base del PUM: los sujetos nulos en los primeros estadios del aprendizaje del inglés como L1 se deben a que, en esos momentos, la flexión verbal es uniforme porque no aparece. En cuanto esta emerge, los sujetos nulos desaparecen.

Acerca de una hipótesis contraria (Hipótesis de la Continuidad Fuerte), según la cual las gramáticas iniciales apenas difieren, en lo que al fenómeno del sujeto nulo se refiere, de las gramáticas adultas, lo que implica no poder asignar un valor no marcado al parámetro, cf. Valian 1991 y 1994, Valian/Eisenberg 1996, Bloom 1991 y Bel 2001, que se centra en el español y el catalán. 
marcado del parámetro permite explicar la mayor facilidad que tienen los aprendices con una L1 en la que no es posible elidir el sujeto para adquirir una L2 en la que sí lo es y las mayores dificultades en la adquisición de una L2 sin sujetos nulos para aprendices cuya L1 sí presenta este tipo de sujetos. ${ }^{5}$ Del mismo modo, las dificultades que entraña el aprendizaje de una L2 en la que este parámetro tiene un valor diferente del que posee la L1 repercuten en la interlengua inicial del aprendiz en el sentido de que los aprendices de una L2 pro-drop cuya L1 no lo es tratarán la primera como si no lo fuera y, al contrario, los aprendices de una L2 que no es pro-drop cuya L1 sí lo es emplearán la L2 como si lo fuera (cf. Phinney 1987, White 1989, 136, Brucart 1998, 29).

Ahora bien, si atendemos a las dos cuestiones que, según White 1989, 86-87, debe adquirir todo aprendiz de una lengua pro-drop -el hecho en sí de que se permitan los sujetos nulos y las restricciones discursivas que gobiernan en esa lengua tanto los sujetos nulos como los explícitos-, que le permitirían no caer en un uso abusivo o en lo contrario, en un uso restringido de uno y otro tipo de sujetos, no parece que la literatura existente al respecto haya aclarado, al menos en lo que se refiere al aprendizaje del español, la segunda de ellas. Es más, parece que la ha dejado explícitamente de lado. Así, por ejemplo, Soler Montes 2002, 34, soslaya el asunto afirmando sorprendentemente que, al aprender español, el uso u omisión del sujeto no es una cuestión de máxima importancia, ya que no afecta al significado (sic) básico de lo que se comunica. Este mismo autor, que reconoce la poca atención que el fenómeno ha recibido en los libros de texto y en las clases de español como L2, aun a pesar de que una gran parte del input que reciben los alumnos en los primeros días de contacto con la lengua española contiene ya sujetos omitidos, ${ }^{6}$ propone que será la repetición constante de ejemplos la manera en la que los estudiantes de L2 podrán reactivar el parámetro y usarlo correctamente. Esto es, ofrece como única solución de enseñanza-aprendizaje exclusivamente la inmersión en el input de la L2, lo que, desde nuestro punto de vista, deja todo sujeto a la pura intuición y degrada en cierto sentido el papel del profesor en el aula. Fernández Fuertes 2004, 8-9, por su parte, postula que los hablantes de inglés aprendices de una L2 con sujetos nulos como el español lo que deberían hacer simplemente es trasladar a la morfología verbal de nuestra lengua las características de los pronombres libres de su lengua materna y entender los sujetos explícitos meramente como sujetos retóricos, duplicados de las desinencias verbales, algo, lo veremos, que dejaría de explicar la casuística que supone la presencia/ausencia del sujeto en español.

No mucho más allá llegan los que han abordado el problema ajenos al modelo de Principios y Parámetros. En este sentido, por ejemplo, Balbino de Amorim Barbieri Durão/Ferreira 2004, 273, que, como muchos otros, siguen pensando que la presencia del sujeto en español cumple, sin más, una función enfática y también

\footnotetext{
5 Acerca de una propuesta distinta, en la que se considera más sencillo pasar de una L1 no marcada a una L2 marcada, cf. White 1985, 1986, 1988 y 1989.

6 Tal y como plantea Soler Montes 2002, 36, es muy frecuente que expresiones del tipo: (1) ¿Cómo te llamas? (2) Me llamo Juan (3) ¿Cuántos años tienes? (4) ¿Cómo estás? surjan en el aula de español L2 en los primeros estadios del aprendizaje.
} 
desambiguadora, advierten de cómo en seis manuales de enseñanza de español para brasileños se obvia una explicación del fenómeno de la presencia/ausencia del sujeto.

Quien sí se ha preocupado por el tema ha sido Matte Bon 2008, 246-251, que, en la undécima reimpresión de su gramática comunicativa de 1992, señala acertadamente que el hecho de que el sujeto pueda elidirse en español no implica que la presencia/ausencia de este se deba al capricho o a la pura casualidad. Sin embargo, cuando aborda la cuestión, partiendo del principio básico de que el sujeto sólo aparece en español cuando al hablante le parece indispensable para la correcta comprensión de sus intenciones comunicativas, se limita a plantear sólo casos en los que el sujeto se encuentra explícito sin distinguir jamás cuándo la presencia es obligatoria y cuándo no lo es. En ningún momento se diferencian los casos en los que la presencia y la ausencia pueden alternar frente a aquellos en los que no, no se hacen distinciones entre casos en los que la alternancia es posible, no se proponen criterios para determinar cuándo la presencia del sujeto es la única opción y lo mismo sucede en lo que atañe a los ejemplos en los que sólo cabe la ausencia.

Desde nuestro punto de vista, un camino para salvar esta situación pasa, tal y como planteábamos en Campos Carrasco/Devís Márquez 2008, por elaborar una reflexión gramatical lo más sencilla posible que permita crear una secuencia explicativa que capacite al aprendiz para inferir el funcionamiento de la presencia/ausencia del sujeto en español y para emplear este conocimiento en situaciones comunicativas determinadas.

\section{LA REFLEXIÓN GRAMATICAL EN EL PROCESO DE ENSEÑANZA- APRENDIZAJE}

Tal y como decíamos en Campos Carrasco/Devís Márquez 2008, una de las grandes polémicas a la hora de abordar los contenidos en los distintos métodos de enseñanza y aprendizaje de lenguas ha sido el de la utilidad de la gramática. Ante el debate de la concienciación metalingüística, debido sobre todo a la falta de paralelismo entre la adquisición de la lengua materna y la de una L2, nos decantábamos por la consideración de la gramática como un instrumento útil. Ahora bien, sólo si se trata de una gramática en un sentido más amplio que el habitual y que abarque aspectos que superen lo estrictamente morfológico y sintáctico esta utilidad será visible. Proponíamos una gramática que, al tiempo que sea comunicativa, esto es, sea entendida como un mero instrumento para la comunicación y no como un objetivo de aprendizaje en sí, también sea útil y asequible para todo tipo de alumno, es decir, pedagógica (cf. Dirven 1990). Una gramática cuyo objeto de estudio sea el uso de los enunciados lingüísticos dentro de contextos concretos de comunicación y que actúe como intérprete entre las habituales gramáticas formales y los aprendices de lenguas extranjeras, que, en un alto porcentaje, carecen de conocimientos gramaticales y no muestran un especial interés por su adquisición.

A la vez, postulábamos como línea de trabajo más efectiva la corriente metodológica basada en el descubrimiento de la gramática por los alumnos, línea a la 
que contribuye de manera decisiva una de las tendencias del método comunicativo: el enfoque mediante tareas, método que planifica las unidades didácticas no sólo mediante tareas comunicativas, sino también mediante tareas intermedias, precomunicativas, posibilitadoras o de contenido gramatical (cf. Estaire/Zanón 1994 y Alba/Zanón 1999). La gramática queda entendida como un medio necesario para poder llevar a cabo las tareas comunicativas y los alumnos van siendo conscientes de ella mediante las tareas intermedias.

Como aporte personal a esta línea de trabajo marcábamos dos objetivos básicos: sentar las bases teóricas y metodológicas para la elaboración de una gramática pedagógica del español y elaborar guías y recursos didácticos en forma de fichas que sirvan de apoyo para que el profesor de español como L2 pueda estructurar las explicaciones de problemas gramaticales del modo más sencillo y práctico. La elaboración de estas fichas didácticas intenta alejarse del molde habitual de "explicación gramatical más batería de ejercicios posterior" y posee como cimientos básicos los conceptos de secuenciación e inferencia: como ya hemos atisbado, a partir de una reflexión gramatical previa que sólo conocerá el profesor se planteará una secuencia explicativa que permita al alumno inferir los conceptos gramaticales que se intentan explicar y que le serán de utilidad en situaciones comunicativas reales. Obviamente, la reflexión gramatical de la que hablamos sólo será -junto con, si fuese necesario, el material adicional previo- el punto inicial de la ficha didáctica. Tras la citada reflexión vendrán, sucesivamente, y por este orden: 1) dependiendo del grado de complicación que suponga la reflexión gramatical, situar la unidad didáctica en uno de los niveles establecidos por el Marco común europeo de referencia para las lenguas (A1, A2, B1, B2, C1, C2). Una opción también puede ser desglosar la unidad didáctica en más de una ficha con distintos niveles de dificultad; 2) los ejercicios de observación, mediante los cuales los alumnos, en pequeños grupos, observarán contextos lingüísticos relacionados con la tarea gramatical de que se trate; 3) los ejercicios de identificación de la regla, de completar secuencias lingüísticas y de repetición de mecanismos, que ayudarán todos a que el aprendiz, en función de lo observado mediante los ejercicios de observación, no sólo decida qué casos son adecuados y cuáles no, sino que también practique con ejemplos concretos. Ello permitirá al profesor reflexionar acerca de la corrección o incorrección de lo observado por el alumno; 4) situaciones comunicativas en el aula, que deben trabajar las cuatro destrezas (comprensión/expresión oral y escrita), y, finalmente, 5) puesta en común como confirmación final de resultados en el aprendizaje en la que, como a lo largo de todo el proceso de enseñanza-aprendizaje, el profesor jugará un papel activocoordinativo y el alumno, uno activo-interactivo.

Nuestro siguiente paso en este artículo, pues, se centra en la elaboración de una reflexión gramatical que sea el punto de partida para crear una ficha didáctica cuyo tema sea, no el de la enseñanza-aprendizaje en el aula de español como L2 del fenómeno de la elisión del sujeto o sujeto nulo, sino el la presencia/ausencia del sujeto en español actual. Las razones son evidentes: en español el sujeto no sólo puede elidirse por razones de tipo discursivo, sino que también habrá casos en los que esta posibilidad de alternar la ausencia junto con la presencia no sea en modo alguno 
aceptable, lo que supondrá bien la presencia bien la ausencia como única opción. En estos casos de no alternancia entre la presencia y la ausencia del sujeto no sólo jugarán un importante papel aspectos discursivos, sino que también lo harán otros de otro tipo: gramaticales y léxicos. Nuestra pretensión de sencillez y utilidad sólo va dirigida al resultado final, esto es, a la propia reflexión gramatical, a la que deben llegar mediante la inferencia alumnos de todo tipo, los que saben gramática y los que no. Lo que sí carece de la obligación de ser sencillo es el proceso descriptivo de la lengua española que nos lleve a esa reflexión gramatical. Partimos de la base de que las descripciones lingüísticas serán tan complicadas -o tan sencillas- como lo sean los fenómenos lingüísticos que se intenta explicar y de que el profesional de la enseñanza de lenguas extranjeras, además del plus pedagógico, tiene que estar tan capacitado para entender y elaborar descripciones lingüísticas como lo deben estar aquellos cuyo objetivo es el estudio de la gramática en sí. De hecho, cuando hablamos de una gramática pedagógica en el aula de L2, estamos pidiendo a este profesional que juegue un papel de intermediario (intérprete o exégeta) entre las gramáticas para gramáticos y estudiantes de gramática y los aprendices de lenguas extranjeras. No obstante, intentaremos que nuestra explicación goce de la mayor claridad y evite en lo posible la abundancia de tecnicismos gramaticales. Cuando el empleo de estos tecnicismos sea imprescindible, remitiremos a referencias bibliográficas concretas que los aclaren.

\section{DESCRIPCIÓN LINGÜÍSTICA Y ELABORACIÓN DE LA REFLEXIÓN GRAMATICAL: PRESENCIA Y AUSENCIA DEL SUJETO EN ESPAÑOL}

En español, la presencia y la ausencia del sujeto con verbos conjugados en forma personal no es -frente a lo postulado por una gran mayoría- una sencilla cuestión de mera distribución contrastiva en el discurso, fundamentalmente porque la alternancia entre una y otra no es posible en todos los casos y porque no siempre que lo es goza de un carácter contrastivo. Puede ser también libre. Cuando no existe alternancia, no una razón concreta, sino una casuística bastante diversa de factores discursivos y gramaticales controlan la presencia del sujeto, en tanto que la ausencia queda al arbitrio no sólo de factores discursivos y gramaticales, sino también, aunque sólo en determinados casos, del contenido léxico de los verbos. ${ }^{7}$ Hablemos en primer lugar de la alternancia entre la presencia y la ausencia del sujeto.

Habrá alternancia libre cuando el hecho de que el sujeto se encuentre explícito o no no suponga modificaciones que tengan que ver con el referente denotado, con la

${ }^{7}$ Tal y como hacemos ahora, es frecuente emplear sin pudor en el aula de L2 la noción de sujeto. Las múltiples dificultades que entraña este concepto gramatical, que no vamos a tratar aquí, pues son objeto de la teoría del lenguaje, no sólo pueden provocar problemas al aprendiz de una L2, sino a los propios estudiantes de la gramática de su lengua materna. Acerca de esta cuestión y una propuesta de solución, cf. Devís Márquez en prensa (b). 
lectura específica o inespecífica de la construcción, con el valor contrastivo o con la modificación del tema discursivo. Así, por ejemplo en:

(5)He quedado con María a las dos. Son las dos y cuarto y todavía (\{Maríalella\}) no ha venido,

se encuentre o no explícito el sujeto de venir, no se producen modificaciones del tipo señalado. Tanto la presencia como la ausencia permiten identificar un mismo referente y la presencia no supone contraste alguno ni entre intervinientes en el suceso que se relata ni entre intervinientes en el acto comunicativo (emisor, receptor o alguien/algo que no sea ni uno ni otro). ${ }^{8}$ Un segundo ejemplo pertenecería al grupo de lo que Bosque 1989, 96 y RAE 2009, 2553, denominan sujetos tácitos argumentales referenciales indefinidos. Nos referimos a un titular de prensa como:

(6) Da a luz en un taxi con la ayuda de la policía

Tal y como plantea la RAE, en casos como este es posible colocar como sujeto del verbo un grupo nominal indefinido. Si bien en otros ejemplos la gama de posibilidades estaría abierta:

(7)(\{Recluso/Delincuente peligroso/Hombre enloquecido\} asesina a inocente y lo inculpa,

en el que proponemos, debido al significado del por algunos llamado predicado complejo dar a luz, la opción es única y sólo permite seleccionar una clase determinada de individuos:

(8) (Mujer) da a luz en un taxi con la ayuda de la policía

De nuevo, tanto la presencia como la ausencia del sujeto permiten identificar a un individuo inespecífico de una clase concreta sin que una u otra impliquen diferencias de contraste, tema discursivo, lectura específica o inespecífica, etc.

Un último ejemplo de alternancia libre: el de las construcciones sin sujeto explícito con el verbo en tercera persona del plural y valor de generalización. En el ámbito de las estructuras oracionales sin sujeto explícito y verbo en tercera persona del plural con lo que algunos han llamado valor genérico convendría distinguir entre lo que en Devís Márquez 2003, 434, denominamos valor de indeterminación (en RAE 2009, 2553, valor inespecífico):

8 Cf. en este sentido la distinción de Enríquez 1984, 128, entre contraposición paradigmática y contraposición sintagmática del pronombre sujeto en español. De manera análoga, Muñoz 1988 distingue entre sujeto enfocado y sujeto foco de contraste. 
(9) Llaman a la puerta (= alguien no identificado y del que se ignora la clase a la que pertenece llama a la puerta)

y valor de generalización (en RAE 2009, 2553-2554, valor genérico):

(10) En España ven demasiado la televisión (= en España todo el mundo, la gente, ve demasiado la televisión).

En estas construcciones con una lectura indefinida no puede alternar la ausencia del sujeto con la presencia sólo en las de indeterminación, pues la inespecificidad en este caso es de los miembros de una clase y de la clase en sí, pero sí en las de generalización, ya que en estas el carácter inespecífico reside sólo en los miembros de la clase a la que se alude, que suele tener una gran amplitud, pero no en la propia clase:

(11) En España los españoles ven demasiado la televisión

Obviamente, la misma alternancia existe cuando estas estructuras con verbo en tercera persona del plural carecen de lectura indefinida:

(12) Desde aquí veo a unos niños. (Los niños) llaman a la puerta y se van

La alternancia o distribución contrastiva entre la presencia y la ausencia del sujeto es clara cuando la primera muestra una lectura de contraste (alguien/algunos frente a otro(s)). Tal contraste sólo se establece entre los intervinientes en el suceso que se relata y nunca entre los intervinientes en el acto comunicativo. Como ejemplos, tres.

(13) Los ladrones actuaron como si estuvieran en su casa, con una frialdad que aún asombra a los investigadores. (Ellos) fueron a la cocina, donde (ellos) se apoderaron de una gran cantidad de fruta que (ellos) sacaron al jardín para comérsela

(14) A casa llegó un grupo de soldados. (Dos de ellos) llamaron enfurecidos a la puerta

(15) Ana tiene coche, pero (yo) voy a trabajar en bus

En el primero, es lícita tanto la presencia como la ausencia de los sujetos de los verbos ir, apoderarse y sacar y tanto en un caso como en otro se mantiene el tema discursivo (los ladrones). La diferencia estriba en que, si los sujetos quedan explícitos, el hablante lo que pretende es establecer un contraste entre los verdaderos autores de las acciones (los ladrones) y otras personas susceptibles de que estas les sean achacadas -imaginemos, por ejemplo, el discurso de un fiscal en un juicio-. Si, por el contrario, están ausentes, este carácter contrastivo desaparece. En el segundo ejemplo, la presencia del sujeto de llamar tiene como intención por parte del hablante 
especificar el número de miembros del grupo que lleva a cabo la acción de llamar frente a los que no llaman. La ausencia, no hacerlo. En el tercero, la presencia implica que, frente a Ana, yo no tengo coche. La ausencia no lo implica, aunque sí la posibilidad de que Ana pudiera llevarme en su coche. El contraste que supone la presencia del sujeto en todos estos ejemplos no viene exigido por el contexto y la situación comunicativos o por la estructura gramatical empleada, sino que es una decisión que libremente toma el hablante.

Cuando la alternancia entre la presencia y la ausencia del sujeto no es factible, disponemos de dos posibilidades: que la presencia sea la única opción o que lo sea la ausencia. En el caso de la presencia obligatoria, esta puede estar motivada bien por razones relacionadas con el contexto y situación que envuelven al discurso, bien por cuestiones gramaticales. Si bien ya hemos visto que determinadas situaciones discursivas facultan al hablante para establecer contraste o no, conviene tener en cuenta que otras le obligan a ello. En este sentido, la presencia obligada por razones discursivas puede estar ocasionada por el hecho de que la situación comunicativa exija al hablante establecer un contraste bien entre los intervinientes en el acto comunicativo, bien entre los intervinientes en el suceso que se relata. Así pues, ante la pregunta:

(16)¿Quién va a pagar?,

el sujeto sólo es obligatorio si contestamos a quien exige el contraste entre los presentes en el acto comunicativo:

(17) $\{$ Yo/Yo pago/Pago yo/*Pago $\}$

La misma situación comunicativa podría dar lugar a que nos dirigiéramos a otro interlocutor distinto del que realiza la pregunta diciendo:

(18) Por favor, (tú) pagas y luego hacemos cuentas,

sin la necesidad ya de hacer explícito el sujeto. Otro ejemplo de claro contraste entre los intervinientes en el acto comunicativo con carácter obligatorio sería cuando alguien nos dice:

(19) No sé qué hacer. Mi jefe me exige que haga horas extraordinarias y no me apetece

y contestamos:

(20) Pues *(yo) no transigiría,

donde la contraposición emisor-receptor resulta evidente. 
Muestra de contraste obligatorio entre los intervinientes en el suceso relatado podría ser:

(21)Me encontré a Juan y Ana por la calle. *(Él) dijo que acababa de nacer su segundo hijo.

Aquí, la presencia obligatoria del sujeto del verbo decir viene dada por la necesidad de establecer un contraste entre Juan y Ana.

La presencia del sujeto puede estar obligada por la existencia de un contraste exigido ahora, no por el contexto y la situación que envuelven al discurso, sino por la propia estructura gramatical que se emplea. En estos casos, el contrate se establece siempre entre intervinientes en el suceso relatado. Nunca entre los que intervienen en el acto comunicativo. Podemos ejemplificar con determinadas estructuras comparativas y con algunas estructuras coordinadas adversativas excluyentes.

En lo que atañe a las comparativas, en español resulta obligatoria la presencia del sujeto en las codas clausales ${ }^{9}$ de este tipo de construcciones en tanto en cuanto este sea el único constituyente en la coda y los verbos de principal y subordinada -el de esta puede no estar explícito- sean el mismo:

(22) Compró tantos libros como (compraste) *(tú)

(23) Pidió más pizzas de las que *(tú) (habias pedido)

Sí puede ser eliminado si, aun siendo los verbos idénticos, el sujeto no es el único en la coda, pues el contraste establecido no se refiere ya en exclusiva a los sujetos de principal y subordinada:

(24) Compró tantos libros como le habías comprado (tú) a tu hermano

(25) Pidió \{más/menos\} pizzas de las que (tú) le habías pedido a tus hijos

Cuando se trata de codas clausales con un verbo diferente del de la principal, la elisión del sujeto es siempre posible:

(26)Juan leyó \{más/menos\} libros de los que (sus padres) le habían comprado (27)Compré tantos vasos como (yo) había pensado ${ }^{10}$

9 Acerca de la distinción entre coda frasal y coda clausal en las estructuras que expresan comparación, cf. Sáez del Álamo 1999, 1132-1133.

10 Incluso, cuando la comparación se centra en los verbos y los sujetos son correferenciales, resulta imposible hacer explícito el de la coda clausal: (28) Juan gasta \{ más/menos\} que (*él) gana; (29) Juan gasta tanto como (*él) gana. No sucede lo mismo si la coda está introducida, en el caso de las comparativas de desigualdad, por "de + relativo no concordante": (30) Juan gasta \{más/menos\} de lo que (él $\left.l_{i}\right)$ gana. Ejemplos como (28) y (29) habría que incluirlos en el apartado de los que muestran la ausencia del sujeto como única opción. 
Los ejemplos (24), (25), (26) y (27) muestran una alternacia contrastiva entre la presencia y la ausencia del sujeto en el sentido expuesto más arriba.

Por su parte, las construcciones adversativas excluyentes -aquellas que implican incompatibilidad entre las dos oraciones coordinadas-, frente a lo que sucede con las no excluyentes, exigen en todas sus variantes la presencia de los sujetos en tanto que el contraste se establezca entre estos. Así, si el contraste tiene que ver con los complementos verbales, la elisión es siempre posible y la presencia/ausencia del sujeto muestra una alternancia contrastiva:

(31) (Juan) quiere la mesa, pero (Juan) no (quiere) la silla

(32) (Juan) no quiere la silla y (Juan) sí (quiere) la mesa

(33) (Juan) no quiere la silla, pero (Juan) sí (quiere) la mesa

(34) (Juan) no quiere la silla, sino que (Juan) (quiere) la mesa

Esto no ocurre cuando el contraste atañe a los sujetos:

(35) Lo hizo *(Juan), pero no (lo hizo) *(Pedro)

(36) *(Juan) lo hizo y no (lo hizo) *(Pedro)

(37) No lo hizo *(Pedro) y sí (lo hizo) *(Juan)

(38) No lo hizo *(Pedro), pero sí (lo hizo) *(Juan)

(39) No lo hizo *(Pedro), sino (que lo hizo) *(Juan)

La situación es diferente cuando se trata de construcciones adversativas no excluyentes, pues en estas la elisión de los sujetos -y, por tanto, la alternancia contrastiva entre ausencia y presencia- es siempre posible estemos ante estructuras ampliativas:

(40) (Juan) nació en Francia, pero (sus padres) lo inscribieron en la embajada española

(41) (Sus padres) lo inscribieron en la embajada española, pero (Juan) nació en Francia

(42) (Juan) nació en Francia y, sin embargo, (sus padres) lo inscribieron en la embajada española,

restrictivas no especificativas:

(43) (Juan) nació en Francia, pero (sus padres) no lo inscribieron allí

(44) (Sus padres) no lo inscribieron allí, pero (Juan) nació en Francia

(45) (Juan) nació en Francia y, sin embargo, (sus padres) no lo inscribieron alli)

o restrictivas especificativas: 
(46) (Juan) quiere un libro, pero (yo) prefiero que sea de Derecho ${ }^{11}$

En lo que concierne a la obligatoriedad del sujeto por razones gramaticales cabe plantearse casos que se explican por la imposibilidad de que la desinencia verbal pueda recuperar toda la información gramatical que supone la presencia del sujeto. Así sucede, por ejemplo, con los cuantificadores indefinidos pronominales:

(47) Nadie sabía nada

(48) Cualquiera le dice algo

(49) Ninguno vino,

con aquellas estructuras en las que el sujeto es un $\mathrm{SN}$ cuantificado cuyo cuantificador posee una variable ligada: ${ }^{12}$

(50) Cada niño pintó dos cuadros,

con el uso genérico del pronombre indefinido uno:

(51) En determinadas ocasiones uno no sabe cómo actuar,

con construcciones interrogativas parciales en las que el pronombre interrogativo es el sujeto:

(52) ¿Quién ha venido?

o con determinados casos de relaciones anafóricas, como el de las construcciones de relativo con antecedente explícito en las que tanto este como el propio pronombre ejercen de sujetos:

(53) El niño que vino ayer a mi casa se llama Juan

y como el de las estructuras que incluyen el neutro ello con antecedente oracional y valor conectivo:

(54) Continúa siendo objetivo común que la línea entre en servicio en el año 2002. Ello implica iniciar las obras en 1996

11 Acerca de la distinción entre coordinadas adversativas excluyentes, no excluyentes restrictivas no especificativas y no excluyentes ampliativas, así como de los criterios para establecerla, cf. Báez San José/Moreno Martínez 1977, 112-119.

12 Acerca de la capacidad de ligar variables de los cuantificadores, cf. Lyons 1977, 147154 y Keenan 1971, 261. 
Con respecto a ejemplos como (47), (48), (49), (50), (51) y (52), habría que decir que ninguno de los contextos posibles en los que podrían aparecer admitiría la conmutación por otros con el sujeto elidido:

(55)*Sabía nada

(56)\#Le dice algo

(57)\#Vino

(58)\#Pintó dos cuadros

(59)\#En determinadas ocasiones sabe cómo actuar

(60) $\#_{¿ H a}$ venido?

Del mismo modo que una expresión como (9), con valor de indeterminación, frente a lo que ocurre con la misma estructura sin lectura indefinida, que sí lo hace, no alterna con ninguna construcción con el sujeto explícito, oraciones como (47), (48), (49), (50), (51) y (52) no alternan con estructuras en las que falta el sujeto del tipo de (55), (56), (57), (58), (59) у (60), algunas de las cuales $-(56),(57),(58),(59)$ у (60) ${ }^{-13}$ sí podrían alternar con otras en las que estuviera ausente la cuantificación indefinida:

(62) El padre le dice algo

(63) Su amigo vino,

la relación entre cuantificador y variable:

(64) Mi hermano pintó dos cuadros,

el valor indefinido genérico:

(65) En determinadas ocasiones Juan sabe cómo actuar

o el carácter parcial de la interogación:

(66) ¿Ha venido Juan?

En el caso de estructuras con relaciones anafóricas, como las construcciones de relativo, es el vínculo tan estrecho entre antecedente y anáfora para que esta reciba su interpretación referencial el que impide la elisión de ambos:

(67) *(El niño) *(que) vino ayer a mi casa se llama Juan

${ }^{13}$ El carácter negativo del existencial indefinido nada exige su aparición en oraciones con valor negativo y, por tanto, ser un término de polaridad negativa, esto es, si bien en (46) la concordancia de polaridad negativa es con nadie, cuando este desaparece, se hace obligatoria la presencia de la negación no (cf. Bosque 1980,19-28 y Sánchez López 1999, 2563 y ss.: (61)*(No) sabe nada. 
En construcciones como (53) la RAE 2009, 2558, advierte de la imposibilidad de suprimir el neutro ello. Argumenta que este pronombre carece de correlato tácito. Desde nuestro punto de vista, la razón de que ello no pueda elidirse es que, más que un carácter contrastivo, ${ }^{14}$ conlleva uno conectivo análogo al de los relativos (cf. Fernández Ramírez ${ }^{2} 1987,68$ ). Tanto es así que cabría una paráfrasis con lo cual:

(69) Continúa siendo objetivo común que la línea entre en servicio en el otoño de 2002, lo cual implica iniciar las obras en 1996

Pasemos, por último, a los casos de no alternancia en los que la ausencia del sujeto surge como opción única. Las razones que ahora justifican esta ausencia obligatoria pueden ser de tipo discursivo, gramaticales o estar relacionadas con el significado léxico del verbo. Las primeras explican, por ejemplo, el uso de estructuras oracionales con formas exclusivas de imperativo en español. ${ }^{15}$ De este modo, si el hablante quiere interpelar enérgicamente al oyente para que actúe de determinada manera, no hace jamás explícito el sujeto $\mathrm{y}$, en todo caso, usa el vocativo. Una madre o padre desesperada(o) porque su(s) hijo(s) no come(n) jamás dirá:

(70)*;Come(d) \{tú/vosotros\} de una vez!,

sino

(71) ¡Come(d) de una vez!

o:

(72) ;Come(d) de una vez, niño(s)!

Sin embargo, si, en otro tipo de situación comunicativa, se quiere atenuar un mandato y convertirlo, casi, en un consejo, nada obsta para que la presencia y la ausencia del sujeto alternen:

(73) \{Come tú/tú come/comed vosotros/vosotros comed\} y \{olvidate/olvidaos\} de los demás

14 Entre las pruebas que aporta Fernández Soriano 1999, 1245, para negar el valor contrastivo de ello se encuentra su incapacidad de aparecer al lado de adverbios que normalmente acompañan al foco oracional: (68)*\{Precisamente/Justamente/Solamente/ Exactamente\} ello implica iniciar las obras en 1996.

${ }^{15}$ Ejarque 1977, 70-71 y 74-75 y Enríquez 1984, 128-129, ya aluden al escaso uso del pronombre personal sujeto en las oraciones imperativas. Según la segunda autora, se trata de construcciones que muestran una gran concreción del sujeto. 
Razones relacionadas con el contexto y situación que rodean a hablante y oyente en el discurso encontramos igualmente en algunas situaciones comunicativas que muestran el polo opuesto de aquellas que exigen al hablante el establecimiento de contraste. Así, si alguien dice:

(74) ;La comida está lista!

con la intención de que determinada(s) persona(s) acuda(n) a comer, la respuesta de esta(s) sería, por ejemplo:

(75) $\{$ Voy/Vamos $\}$,

pero jamás:

(76) \{\#Yo voy/\#Voy yo/\#Nosotros vamos/\#Vamos nosotros\},

fundamentalmente porque quien dio el aviso de llamada tiene ya un conocimiento previo de quién va a responder y de quién va a ir, tanto es así que la respuesta por parte del (de los) interlocutor(es) a quien(es) se da aviso podría ser con la tercera persona -la no persona- sin que ello supusiese ambigüedad alguna:

(77) $V a$

Un último ejemplo de ausencia obligatoria del sujeto debida a razones meramente comunicativas puede ser el de lo que algunos han denominado subordinadas relativas predicativas dependientes de un verbo de percepción física:

(78) Oigo a María que canta

(79)*Oigo a María que ella canta

Que en estructuras como la del ejemplo (77) la subordinada no modifica al nominal expreso María -cuya denotación no puede ser restringida- lo demuestra el hecho de que ese elemento nominal pueda ser sustituido por un clítico y que la pronominalización deje fuera la subordinada:

\section{(80) La oigo que canta}

Por consiguiente, resulta obvio que el relativo que no tiene como antecedente el nombre propio María (cf. Devís Márquez 2009, 452). Ahora bien, ya en Devís Márquez en prensa (a) señalábamos que, desde el momento en que las subordinadas de construcciones como la que ahora tratamos no tienen como antecedente el elemento nominal explícito ni admiten sustitución pronominal alguna:

(81)*Lo oigo a María, 
resulta más acertado explicarlas como relativas libres (cf. Brucart 1999a, 445 y ss.) con un antecedente elíptico con valor temporal:

(82) Oigo a María (en el momento en) que canta

Nuestro análisis se ve refrendado por el hecho de que estas construcciones sólo son posibles cuando el verbo de la principal y el de la subordinada coinciden temporalmente (cf. Brucart 1999a, 432, n. 35):

\section{(83)*Oigo a María que \{cantó/había cantado\})}

Si se observa, que se haga explícita la secuencia elidida en estos ejemplos de relativas libres implica que la alternancia entre la ausencia y la presencia del sujeto de la subordinada deja de ser imposible:

(84) Oigo a María $a_{i}$ en el momento en que (ella $a_{i}$ canta

Sí lo es cuando tal secuencia no aparece, pensamos, porque la ausencia de la preposición ante el relativo puede llevar a que este -como de hecho suele hacerse- se interprete como anáfora cuyo antecedente es el nominal explícito, en nuestro ejemplo, María, y, por tanto, como sujeto de la subordinada. Que los relativos, en condiciones normales, no suelan admitir la reduplicación de su propia función mediante un pronombre pleonástico o reasuntivo (cf. Brucart 1999a, 403 y ss.) puede hacer pensar que, en casos como este, el verdadero sujeto no aparece para impedir la reduplicación del pronombre relativo.

Que todo esto es así lo corrobora el que con la secuencia en el momento en elidida el verbo de la subordinada debe concordar siempre en número y persona con el OD de la principal:

\section{(85)*Oigo a María que \{canto/cantas/cantamos/cantáis/cantan\}}

Sin embargo, con la secuencia explícita, desde el momento en que el relativo no se cataloga como sujeto de la subordinada correferente con el OD de la principal, esa concordancia ya no es obligatoria y cabe la alternancia entre la presencia y la ausencia del sujeto:

(86) Oigo a Maria en el momento en que \{(los niños) cantan/(tú) cantas/(Juan) canta\}.

${ }^{16}$ Planteamiento como el de Suñer 1978 y 1982 (cf. Bosque 1989, 95, n. 11), según el cual este tipo de relativas llamadas predicativas son casos de construcciones con sujeto tácito o nulo obligatoriamente correferente con el nominal OD de la principal, no sólo deja sin resolver la función del relativo en las construcciones con verbos de percepción física, sino que tampoco daría cuenta de que en otras también denominadas relativas predicativas esa 
Por tanto, es el factor discursivo de la elisión el que determina la existencia o no de la alternancia. ${ }^{17}$

Entre los casos en los que la ausencia obligatoria del sujeto se debe a causas gramaticales cabe citar las estructuras con verbo en tercera persona del plural que expresan indeterminación (cf. ejemplo (9)), las codas clausales, no introducidas por "de + relativo no concordante", de las construcciones comparativas en las que la comparación se centra en los verbos y los sujetos son correferenciales (cf. ejemplos (28) y (29)), las llamadas impersonales con se (impersonales reflejas), etc. En estas últimas, del mismo modo que en las construcciones sin sujeto y verbo en tercera persona del plural, resulta posible distinguir entre aquellas que tienen valor de indeterminación:

(97) Se agredió a una periodista de TVE

(98) Se trabaja intensamente en ello

(99) Cerca de mi casa se vende botellas

y las que lo poseen de generalización:

(100) En Cuba se vive muy bien

(101) En mi pueblo se cubre las casas con tejas,

aunque jamás indican la no indefinición. Frente a lo que ocurre con las construcciones sin se y verbo en tercera persona del plural, que, lo hemos visto, pueden alternar la ausencia y la presencia del sujeto cuando expresan bien no indefinición, bien generalización, las llamadas impersonales con se no gozan de esta opción ni siquiera cuando señalan generalización: ${ }^{18}$

correferencia no es obligatoria, lo que permite, incluso, explicitar el sujeto de la subordinada: (87) Lo dejaron que (sus padres) no lo reconocieron; (88) Lo dejaron que (Juan) no supo qué decir; (89) Los hay que (sus padres) no los quieren en casa; (90) Los hay que ni (ellos) saben qué hacer).

${ }^{17}$ En el caso de las construcciones con verbo de percepción física, si el OD del verbo principal no fuese un nombre propio: (91) Oigo las campanas que suenan, desde el momento en que ya sí es posible restringir la denotación del sustantivo, no sólo cabe interpretar en todos los sentidos la subordinada como en el ejemplo con nombre propio: (92) Las oigo que suenan, sino también como relativa especificativa. Esta segunda opción permite que la pronominalización del OD englobe a la subordinada: (93) Las oigo y no exige la coincidencia temporal entre los verbos de principal y subordinada: (94) Oigo las campanas que \{sonaron/habian sonado\}. En cuanto a la presencia del sujeto de la subordinada, esta sólo será obligatoria si tal sujeto es el mismo relativo: (95) Oigo las campanas *(que) \{suenan/habian sonado\}, pero será facultativa si el sujeto está representado por cualquier otro elemento distinto: (96) Oigo las campanas que (unos amigos) habian escuchado.

${ }^{18}$ En Devís Márquez en prensa (b), donde no hablamos de sujeto como función sintáctica oracional, sino de constituyente determinado por el verbo, proponemos situar las denominadas pasivas con se (pasivas reflejas): (102)Se escribieron los documentos en el nuevo ordenador; 
(110)*En Cuba toda la gente se vive muy bien

(111)*En mi pueblo la gente se cubre las casas con tejas

Razones relacionadas con el contenido léxico del verbo en lo que concierne a la ausencia obligatoria del sujeto explican las construcciones con verbos -por algunos llamados impersonales léxicos- que no varían en cuanto a la persona y el número:

(112) Llueve $e^{19}$

(114) Hace frio

(115) Hay moscas en el jardín

(116) Es tarde

Se trata de verbos cuyas características semánticas impiden modificar la denotación de cualquier otro elemento oracional. ${ }^{20}$ Ahora bien, en español hay construcciones con el verbo ser, habitualmente catalogadas como estructuras con sujeto, a las que algunos autores denominan impersonales que realizan juicios esenciales, las cuales, dicen, predican sólo la presencia de una entidad, no su existencia, y se parafrasean bien con se trata de:

(103)Se reordenó el tráfico para que pasara la comitiva; (104)En España se comen muchas naranjas, junto a las impersonales reflejas. Nuestro argumento principal es la carencia en ambas del citado constituyente determinado por el verbo, si bien no es este un asunto para ser tratado aquí.

Sin embargo, no incluimos en este grupo que expresan indeterminación y generalización otras construcciones con se en las que o bien aparece un tradicional sujeto que sufre un proceso agentivo y el pronombre reflejo varía en cuanto a la persona a la vez que lo hace el propio sujeto: (105)La casa se quemó accidentalmente; (106)Yo me quemé accidentalmente; (107)Tú te quemaste accidentalmente, o bien encontramos un sujeto cuya denotación es modificada explicándola, esto es, atribuyéndole una cualidad inherente, y no resulta posible la variación del pronombre reflejo (las llamadas medias-pasivas (cf. Mendikoetxea 1999, 1641): (108)Esta camisa se lava muy bien. Obviamos la descripción de estructuras con se y complementos introducidos por la preposición por: (109)??Se firmó la paz por los embajadores, que, más que atestiguadas por el uso, parecen estarlo por determinados gramáticos.

${ }^{19}$ Un verbo como llover no varía en cuanto a la persona y el número cuando expresa fenómeno meteorológico, pero puede mostrar variación en lo que respecta al número cuando presenta un significado léxico análogo al de caer (113) Llueven piedras

${ }^{20}$ A las construcciones en las que aparece este tipo de verbos Bosque 1989, 97, Brucart 1999b, 2851 y la RAE 2009, 2554, les asignan lo que denominan sujetos tácitos o nulos no argumentales, que equivalen, dicen, a los sujetos expletivos de otras lenguas: (117) Il pleut; (118) It rain; (119) Es regnet. En este sentido, la RAE 2009, 2554, señala cómo en el español popular de la República Dominicana aparecen construcciones del tipo: (120) Ello está lloviendo; (121) Ello hacía calor, lo que demuestra, dice, que los sujetos expletivos no son desconocidos en español. Acerca de otro punto de vista, cf. Devís Márquez en prensa (b). 
(122) Soy yo

(123) Somos nosotros

(124) Es Juan,

bien con se da esa circunstancia:

(125)Es que me canso

(126)Son las cuatro

(127)Es la una ${ }^{21}$

De nuevo, las características semánticas del verbo impiden la modificación de la denotación del constituyente que le acompaña. Ello queda reflejado en la imposibilidad de dislocarlo a la izquierda y situarlo delante del verbo, algo siempre posible con el sujeto: ${ }^{22}$

$(133) *$ Yo soy

(134)*Nosotros somos

(135)*Juan es

$(136)^{*}$ Que me canso es

(137)*Las cuatro son

$(138)^{*}$ La una es

${ }^{21}$ Cf. Bosque 1989, 98-101 y 105-106, que cataloga estas construcciones como estructuras con sujeto nulo no argumental análogo a los expletivos de lenguas sin sujetos nulos: (128) C'est moi; (129) It's me; (130) Das bin ich, en las que unidades que carecen de valor indeterminado (yo, nosotros, Juan, que me canso, las cuatro, la una) se consideran parte del SV. Sobre nuestro punto de vista, cf. Devís Márquez en prensa (b), n. 43.

22 Una construcción como: (131) Son tus amigos, sería ambigua, por estar entre el valor impersonal (con sujeto nulo no argumental equivalente a los expletivos) y el copulativo (con sujeto tácito argumental) (cf. Bosque 1989, 100-101),. Sólo en la segunda interpretación podría alternar con una construcción con el sujeto explícito: (132) Ellos son tus amigos Acerca de nuestra opinión, cf. P. P. Devís Márquez en prensa (b): n.44. 


\section{RESULTADO Y CONCLUSIÓN}

Todo lo anterior no es información para el alumno de español como L2, sino para el docente, y puede esquematizarse del siguiente modo, de manera que se convierta en una sencilla reflexión gramatical punto de partida de la(s) ficha(s) didáctica(s):

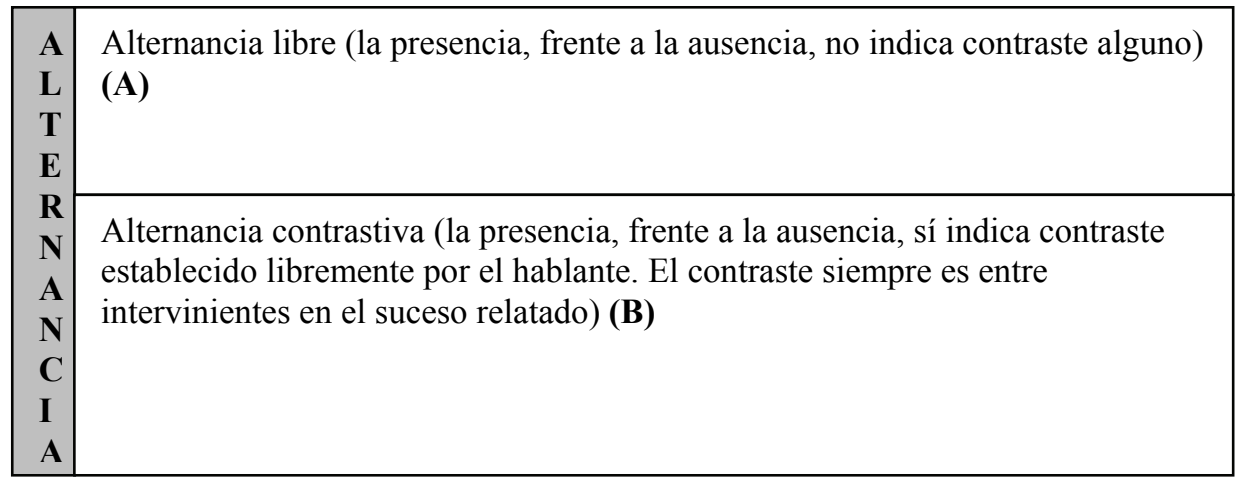




\begin{tabular}{|c|c|c|c|}
\hline \multirow{4}{*}{$\begin{array}{l}\mathbf{N} \\
\mathbf{O}\end{array}$} & \multirow{4}{*}{$\begin{array}{l}\text { La presencia } \\
\text { como única } \\
\text { opción }\end{array}$} & \multirow{2}{*}{$\begin{array}{l}\text { Contraste exigido por } \\
\text { razones discursivas }\end{array}$} & $\begin{array}{l}\text { Contraste entre intervinientes } \\
\text { en el acto comunicativo (C) }\end{array}$ \\
\hline & & & $\begin{array}{l}\text { Contraste entre intervinientes } \\
\text { en el suceso relatado (D) }\end{array}$ \\
\hline & & \multirow[t]{2}{*}{ Causas gramaticales } & $\begin{array}{l}\text { Contraste exigido por la } \\
\text { estructura gramatical (siempre } \\
\text { entre intervinientes en el suceso } \\
\text { relatado) (E) }\end{array}$ \\
\hline & & & $\begin{array}{l}\text { La desinencia verbal no puede } \\
\text { recuperar la información } \\
\text { gramatical que supone la } \\
\text { presencia del sujeto }(\mathbf{F})\end{array}$ \\
\hline \multirow{8}{*}{$\begin{array}{l}\mathbf{A} \\
\mathbf{L} \\
\mathbf{T} \\
\mathbf{E} \\
\mathbf{R} \\
\mathbf{N} \\
\mathbf{A} \\
\mathbf{N} \\
\mathbf{C} \\
\mathbf{I} \\
\mathbf{A}\end{array}$} & \multirow{8}{*}{$\begin{array}{l}\text { La ausencia } \\
\text { como única } \\
\text { opción }\end{array}$} & \multirow{3}{*}{ Causas discursivas } & $\begin{array}{l}\text { Interpelación enérgica al oyente } \\
\text { con imperativo (G) }\end{array}$ \\
\hline & & & $\begin{array}{l}\text { Conocimiento previo del } \\
\text { referente por parte del oyente } \\
\text { (H) }\end{array}$ \\
\hline & & & Elipsis (I) \\
\hline & & \multirow{3}{*}{ Causas gramaticales } & $\begin{array}{l}\text { Estructuras con verbo en } \\
\text { tercera persona del plural que } \\
\text { expresan indeterminación (J) }\end{array}$ \\
\hline & & & $\begin{array}{l}\text { Codas clausales, no introducidas por } \\
\text { "de + relativo no concordante", de } \\
\text { las construcciones comparativas en } \\
\text { las que la comparación se centra en } \\
\text { los verbos y los sujetos son } \\
\text { correferenciales }(\mathbf{K})\end{array}$ \\
\hline & & & Impersonales con se (L) \\
\hline & & \multirow{2}{*}{ Causas léxicas } & $\begin{array}{l}\text { Estructuras con verbos } \\
\text { impersonales léxicos } \mathbf{( M )}\end{array}$ \\
\hline & & & $\begin{array}{l}\text { Impersonales con ser que } \\
\text { expresan juicios esenciales (N) }\end{array}$ \\
\hline
\end{tabular}


Ejemplos:

(A) (5), (6), (8), (10), (11), (12)

(B) ((13), (14), (15), (24), (25), (26), (27), (30), (31), (32), (33), (34), (40), (41), (42),

(43), (44), (45), (46), (62), (63), (64), (65), (66), (82), (84), (86), (87), (88), (89), (90),

(96)

(C) (17), (20)

(D) $(21)$

(E) (22), (23), (35), (36), (37), (38), (39)

(F) (16), (47), (48), (49), (50), (51), (52), (53), (54), (69), (95)

(G) $(71),(72)$

(H) (75), (77)

(I) (78), (80)

(J) (9)

(K) (28), (29)

(L) (97), (98), (99), (100), (101)

(M) (112), (114), (115), (116)

(N) (122), (123), (124), (125), (126), (127)

Como conclusión, parece que para un correcto proceso de enseñanza-aprendizaje en el aula de español L2 resulta indispensable desechar la propuesta de que la lengua española es una lengua pro-drop prototípica y, por consiguiente, la de centrar ese proceso, en lo que atañe al comportamiento del sujeto, exclusivamente en el fenómeno de la elisión de este. Mejor hacerlo en uno más amplio que contemple tanto la ausencia como la presencia en todos sus aspectos. Por último, que el nivel de dificultad parezca menor en todo aquello que tiene que ver con la alternancia entre la presencia y la ausencia del sujeto en español y que esta alternancia se muestre como el caso más frecuente hace recomendable establecer dos etapas en la enseñanzaaprendizaje: primero, la alternancia; con posterioridad, la no alternancia.

\section{REFERENCIAS BIBLIOGRÁFICAS}

ALBA, J. M./ZANÓN, J. (1999): "Unidades didácticas para la enseñanza del español/LE en los Institutos Cervantes", en: Zanón, J. (coord.), La enseñanza del español mediante tareas, Madrid, Edinumen, 149-171.

BÁEZ SAN JOSÉ, V./MORENO MARTÍNEZ, M. (1977): "La oración compuesta I: coordinación", Millars 4, 91-129.

BALBINO DE AMORIM BARBIERI DURÃO, A./FERREIRA, C. C. (2005): "Los pronombres personales sujeto en libros didácticos de español como lengua extranjera", Actas del XV Congreso Internacional de ASELE. Las gramáticas y los diccionarios en la enseñanza del español como segunda lengua: deseo y realidad, Sevilla, Universidad de Sevilla, 273-279. 
BEL, A. (2001): "Sujetos nulos y sujetos explícitos en las gramáticas iniciales del castellano y el catalán", Revista Española de Lingüística 31, 2, 537-561.

BERWICK, R. (1985): The acquisition of syntactic knowledge, Cambridge, Mass., MIT Press.

BLOOM, P. (1991): "Subjectless sentences in child language", Linguistic Inquiry 21, 491-504.

BLOOM, P./LIGHTBOWN, P./HOOD, L. (1975): Structure and variation in child language, Monographs of the Society for Research in Child Development, 40.

BORER, H. (1984): Parametric syntax: case study in Semitic and Romance languages, Dordrecht, Foris.

BOSQUE, I. (1980): Sobre la negación, Madrid, Cátedra.

BOSQUE, I. (1989): "Clases de sujetos tácitos", en: Borrego Nieto, J./Gómez Asencio, J. J./Santos Río, L. (eds.), Philologica II. Homenaje a D. Antonio Llorente, salamanca, ediciones Universidad de Salamanca, 91-111.

BROWN, R. (1973): A first language: the early stages, Cambridge, Mass., Harvard University Press.

BRUCART, J. M" (1998): "Gramática y adquisición en la enseñanza del español como lengua extranjera", en: Celis, A./Heredia, R. (coords.), Lengua y cultura en la enseñanza del español a extranjeros, Cuenca, Ediciones de la Universidad de Castilla-La Mancha, 17-43.

BRUCART, J. Ma . (1999a): "La estructura del sintagma nominal: las oraciones de relativo", en: Bosque, I./Demonte, V. (dir.), Gramática descriptiva de la lengua española, Madrid, Espasa, 395-522.

BRUCART, J. Ma (1999b): "La elipsis", en: Bosque, I./Demonte, V. (dir.), Gramática descriptiva de la lengua española, Madrid, Espasa, 2787-2863.

BURZIO, L. (1986): Italian syntax, Dordrecht, Reidel.

CAMACHO, J. (2010): "El parámetro del sujeto nulo como un caso de movimiento", Cuadernos de la ALFAL 1, 83-93.

CAMPOS CARRASCO, N./DEVÍS MÁRQUEZ, P. P. (2008): "Sobre los contenidos gramaticales en el aula de lengua extranjera", Verbum. Analecta Neolatina 10, 2,485-503.

CONSEJO DE EUROPA (2002): Marco común europeo de referencia para las lenguas: aprendizaje, enseñanza, evaluación, Madrid, Servicio de Publicaciones del Ministerio de Educación, Cultura y Deporte (2001 por Cambridge University Press, con el título Common European framework of Reference for Languages: learning, teaching, assessment).

CHOMSKY, N. (' 1982$)$ : Lectures on government and binding, Dordrecht, Foris.

CHOMSKY, N. (1986): Knowledge of language: its nature, origin, and use, New York, Praeger.

CHOMSKY, N. (1988a): La nueva sintaxis. Teoría de la rección y el ligamiento, Barcelona, Paidós.

CHOMSKY, N. (1988b): Language and problems of knowledge, Cambridge, Mass., MIT Press. 
CHOMSKY, N. (1992): “A minimalist program for linguistic theory”, MIT Ocasional Papers in Linguistics, Cambridge, Mass., The MIT Press.

CHOMSKY, N. (1995): The Minimalist Program, Cambridge, Mass., The MIT Press.

CHOMSKY, N./LASNIK, H. (1993): "The theory of Principles and Parameters", en: J. Jacobs, A. von Stechow, W. sternfled, T. Venneman (eds.), Syntax: an international handbook of contemporary research, vol. 1, Berlin, de Gruyter, 506509.

DEVÍS MÁRQUEZ, P. P. (2003): "La impersonalidad y las denominadas construcciones impersonales en español", Zeitschrift für romanische Philologie $119,3,393-442$.

DEVÍS MÁRQUEZ, P. P. (2009) "Determinación y complementación del nombre propio en español", Nueva Revista de Filología Hispánica 57, 2, 441-488.

DEVÍS MÁRQUEZ, P. P. (en prensa (a)): "Estructuras de control con verbos de percepción en español", Revista Internacional de Lingüistica Iberoamericana.

DEVÍS MÁRQUEZ, P. P. (en prensa (b)):"Presencia y ausencia del sujeto con verbos en forma personal en español", Zeitschrift für romanische Philologie.

DIRVEN, R, (1990): "Pedagogical grammar", Language Teaching 23,1-18.

EJARQUE, D. (1977): "El pronombre personal sujeto en español", Cuadernos de Filología 7, 29-85.

ENRÍQUEZ, E. V. (1984): El pronombre personal sujeto en la lengua española hablada en Madrid, Madrid, CSIC, Instituto Miguel de Cervantes.

ESTAIRE, S./ZANÓN, J. (1994): Planning classwork: a task-based approach, Oxford, Heinemann.

FERNÁNDEZ FUERTES, R. (2004): "La teoría lingüística y la comparación de las lenguas: el caso de los sujetos del español y del inglés", Red ELE. Revista Electrónica de Didáctica/Español Lengua Extranjera 0.

FERNÁNDEZ RAMÍREZ, S. ( $\left.{ }^{2} 1987\right)$ : Gramática española. El pronombre, volumen preparado por José Polo, Madrid, Arco Libros.

FERNÁNDEZ SORIANO, O. (1999): "El pronombre personal. Formas y distribuciones. Pronombres átonos y tónicos", en: Bosque, I./Demonte, V. (dir.), Gramática descriptiva de la lengua española, Madrid, Espasa, 1209-1273.

GUILFOYLE, E. (1984): "The acquisition of tense and the emergence of lexical subjects", The McGill Working Papers in Linguistics, 9, 121-145.

HUANG, C. T. J. (1984): "On the distribution and reference of empty pronouns", Linguistic Inquiry 15, 531-574.

HYAMS, N. (1983): The acquisition of paramaterized grammars, Doctoral dissertation, New York, City University of New York.

HYAMS, N. (1986): Language acquisition and the theory of parameters, Dordrecht, Reidel.

HYAMS, N. (1992): "A reanalysis of null subjects in child language", en: Weissenborn, J./Goodluck, H./Roeper, T. (eds.), Theoretical issues in language acquisition, Hillsdale, Lawrence Erlbaum.

JAEGGLI, O. A. (1980): On some phonologically null elements in syntax, Doctoral dissertation, Cambridge, Mass., MIT. 
JAEGGLI, O. A./HYAMS, N. (1988): "Morphological uniformity and the setting of null subject parameter", Proceedings of the North Eastern Linguistic Society 18, Amherst, University of Massachusetts, 238-253.

JAEGGLI, O. A./SAFIR, K. J. (eds.) (1989): The null subject parameter, Dordrecht, Kluwer Academic Publishers.

JAEGGLI, O. A./SAFIR, K. J. (1989): "The null subject parameter and parametric theory", en: Jaeggli, O. A./Safir, K. J. (eds.), The null subject parameter, Dordrecht, Kluwer Academic Publishers, 1-44.

LAKSHMANAN, U. (1994): Universal Grammar in child second language acquisition, Amsterdam, John Benjamins.

KEENAN, E. L. (1971): "Quantifier structures in English", Foundations of Language 7, 255-284.

LEBEAUX, D. (1988): Language acquisition and the form of grammar, tesis doctoral, Amherst, University of Massachusetts.

LICERAS, J. M. (1994): "La teoría gramatical y los principios que regulan la adquisición del orden de palabras en español", Primera Mesa Redonda de Lingüistica Española, México, Universidad Autónoma Metropolitana de México.

LICERAS, J. M. (1995): "Los procesos de selección, instrucción y representación en la adquisición del lenguaje no nativo", Didáctica 7, 187-202.

LICERAS, J. M. (1996): La adquisición de las lenguas segundas y la gramática universal, Madrid, Síntesis.

LICERAS, J. M./DÍAZ, L. (1999): "Topic-drop versus pro-drop: null subjects and pronominal subjects in the Spanish L2 of Chinese, English, French, German, Japanese and Korean speakers", Second Language Research 15, 1, 1-40.

LICERAS, J. M./DÍAZ, L. (2000): "La teoría chomskiana y la adquisición de la gramática no nativa: a la búsqueda de desencadenates", en: C. Muñoz (ed.), Segundas lenguas. Adquisición en el aula, Barcelona, Ariel, 39-80.

LICERAS, J. M./DÍAZ, L./MAXWELL, D. (1998): "Null arguments in non-native grammars: the Spanish L2 of Chinese, English, French, German, Japanese and Korean speakers", en: Klein, E./Martohardjono (eds.), The development of second language grammar. A generative approach, Amsterdam, John Benjamins, 113149.

LICERAS, J. M./LAGUARDIA, B./FERNÁNDEZ, R./FERNÁNDEZ, Z./DÍAZ, L. (1998): "Licensing and identification of null categories in Spanish non-native grammars", en: Lema, J.//Treviño, E. (eds.), Theoretical analysis of Romance languages, Amsterdam, John Benjamins, 263-282.

LICERAS, J. M./VALENZUELA, E./DÍAZ, L. (1999): "L1 and L2 developing Spanish grammars and the "pragmatic deficit hipótesis"”, Second Language Research 15, 2, 161-190.

LILLO-MARTIN, D. (1991): Universal Grammar and American signlanguage, Dordrecht, Kluwer Academic Publishers.

LU, H.-Ch. (1997): "La presencia y ausencia del pronombre personal", EPOS 13, 117-133.

LYONS, J. (1977): Semantics, vol. 1, Cambridge, Cambridge University Press. 
MENDIKOETXEA, A. (1999): "Construciones con se: medias, pasivas e impersonales", en: Bosque, I./Demonte, V. (dir.), Gramática descriptiva de la lengua española, Madrid, Espasa, 1631-1722.

MOHANAN, K. P. (1983): "Functional and anaphoric control", Linguistic Inquiry 14, 641-674.

MUÑOZ, C. (1988): "La presencia obligatoria del sujeto pronominal en inglés y en castellano", Atlantis 10, 1-2, 37-44.

PERLMUTTER, D. (1971): Deep and surface structure constraints in syntax, New York, Holt, Rinehart and Winston.

PHINNEY, M. (1987): “The pro-drop parameter in second language acqisition", en: Roeper/T./Williams, E. (eds.), Parameter setting, Dordrecht, Reidel, 221-238.

RAE (2009): Nueva gramática de la lengua española. Sintaxis II, Madrid, Espasa Libros, S. L. V.

RIZZI, L. (1982): Issues in Italian syntax, Dordrecht, Foris.

SAFIR, K. J. (1982): Syntactic chains and the definiteness effect, Doctoral dissertation, Cambridge, Mass., MIT.

SÁEZ DEL ÁLAMO, L. A. (1999): "Los cuantificadores: las construcciones comparativas y superlativas", en: Bosque, I./Demonte, V. (dir.), Gramática descriptiva de la lengua española, Madrid, Espasa, 1129-1188.

SÁNCHEZ LÓPEZ, C. (1999): "La negación", en: Bosque, I./Demonte, V. (dir.), Gramática descriptiva de la lengua española, Madrid, Espasa, 2561-2634.

SOLER MONTES, C. (2002): "Principios y parámetros en la adquisición del español como lengua extranjera: el parámetro del sujeto nulo", Cuadernos Cervantes de la Lengua Española 8, 39, 33-36.

SUÑER, M. (1978), "Perception verb complements in Spanish: same or different", Canadian Journal of Linguistics 23, 107-127.

SUÑER, M. (1982): "On null subjects", Linguistic Analysis 9, 1, 55-78.

SUÑER, M. (1984): "Controlled pro", P. Baldi (ed.): Papers from the 12th Linguistic Symposium on Romance Languages, Amsterdam, John Benjamins, 253-273.

VALIAN, V. (1991): "Syntactic subjects in the early speech of American and Italian children", Cognition 40, 21-82.

VALIAN, V. (1994): "Children's postulation of null subjects: parameter setting and language acquisition", en: Lust, B./Hermon, G./Kornfilt, J. (eds.), Syntactic theory and first language acquisition: cross-linguistic perspectives. Vol. 2. Binding, dependencies, and learnability, Hove-London, Lawrence Erlbaum Associates Publishers, 273-286.

VALIAN, V./EISENBERG, Z. (1996): "The development of syntactic subjects in Portuguese-speaking children", Journal of Child Language 23, 1, 103-208.

VANIKA, A./LEVY Y. (1999): "Empty subjects in Finnish and Hebrew", Natural Language and Linguistic Theory 17, 613-671.

VANIKA, A./LEVY, Y. (2000): "The development of a mixed null subject system: a cross-linguistic perspective with data on the acquisition of Hebrew", Language Acquisition 8, 4, 363-384. 
WANG, Q./LILLO-MARTIN, D./BEST, C./LEVITT, A. (1992): "Null subjects and objects in the acquisition of Chinese", Language Acquisition 2, 221-254.

WEXLER, T./MANZINI, R. (1987): "Parameters and learnability in binding theory", Roeper, T./Williams, E. (eds.), Parameter-setting, Dordrecht, Reidel.

WHITE, L. (1985): "The pro-drop parameter in adult second language acquisition", Language Learning 35, 47-62.

WHITE, L. (1986): "Markedness and parameter setting: some implications for a theory of adult second language acquisition", en: Eckman, F./Moravscik, E./Wirth, J. (eds.), Markedness, Markedness, Plenum Press.

WHITE, L. (1988): "Universal Grammar and language transfer", en: Pankhurst, J./Sharwood Smith, M./van Buren, P. (eds.), Learnability and second language: a book of readings, Dordrecht, Foris

WHITE, L. (1989): Universal Grammar and second language acquisition, Amsterdam, John Benjamins. 